\title{
Erosion of the inner magnetosphere during geomagnetic storms
}

\author{
E. Y. Feshchenko ${ }^{1}$, Y. P. Maltsev ${ }^{2}$ \\ ${ }^{1}$ Physical Department, Saint-Petersburg University, Saint-Petersburg, 198904, Russia \\ 2 Polar Geophysical Institute, Apatity, 184200, Russia; e-mail address: maltsev@pgi-ksc.murmansk.su.
}

Received: 4 January 1996 / Revised: 4 June 1997 / Accepted: 25 June 1997

\begin{abstract}
Using the empirical magnetic field model dependent on the Dst index and solar wind dynamic pressure, we calculated the behaviour of the contour $B=B_{s}$ in the equatorial plane of the magnetosphere where $B_{s}$ is the magnetic field in the subsolar point at the magnetopause. The inner domain of the magnetosphere outlined by this contour contains the bulk of geomagnetically trapped particles. During quiet time the boundary of the inner magnetosphere passes at the distance $\sim 10 \mathrm{R}_{\mathrm{E}}$ at noon and at $\sim 7 \mathrm{R}_{\mathrm{E}}$ at midnight. During very intense storms this distance can be reduced to $4-5 R_{E}$ for all MLT. The calculation results agree well with the satellite measurements of the magnetopause location during storms. The ionospheric projection of the $B=B_{s}$ contour calculated with the Euler potential technique is close to the equatorward edge of the auroral oval.
\end{abstract}

\section{Introduction}

The magnetosphere may be provisionally divided into two domains: the inner and the outer ones. For their boundary in the equatorial plane it is natural to choose the contour $B=B_{s}$ where $B_{s}$ is the magnetic field in the subsolar point at the magnetopause. Arykov and Maltsev (1996) and Maltsev et al. (1996) have shown that the geomagnetic storm time depression is determined mainly by the magnetic flux beyond this boundary.

The contour $B=B_{s}$ is also of interest because the bulk of energetic trapped particles is concentrated in the inner domain outlined by it. The outer boundary of the

Correspondence to: Y. P. Maltsev stable trapping does not coincide precisely with this contour due to a splitting of the drift shells in the azimuthally asymmetrical magnetic field. Roederer (1967) has calculated the splitting in the Mead (1964) magnetic field model and has shown, for instance, that if the start point at the noon meridian is located at a geocentric distance of $9 R_{E}$ then the particles with the equatorial pitch-angles $\alpha_{\mathrm{e}}=0^{\circ}$ at midnight are the distance of $\sim 10 \mathrm{R}_{\mathrm{E}}$ whereas the particles with $\alpha_{\mathrm{e}}=78^{\circ}$ are at $\sim 7 \mathrm{R}_{\mathrm{E}}$. The particles with $\alpha_{\mathrm{e}}=90^{\circ}$ drift along those points of the magnetic field lines where the magnetic field is minimum. Far from the magnetopause this corresponds to the contours $B=$ const in the equatorial plane (providing there is no dipole tilt). Near the magnetopause the $B$-minimum surface bifurcates into two separate sheets, one sheet being in the Northern Hemisphere, the other one in the Southern Hemisphere (Shabansky, 1971). For the model by Mead (1964), the thickness of the bifurcation region mapped to the equatorial plane is about $2 \mathrm{R}_{\mathrm{E}}$ (Schulz, 1975).

Since the spatial scales of the splitting and bifurcation regions are not too large compared to the size of the inner magnetosphere the contour $B=B_{s}$ may be roughly considered as the outer boundary of the stable trapping. In the course of disturbances the size of the inner magnetosphere varies more than its shape so that the real trapping boundaries are "tied" to this contour and vary in the same manner.

For quiet conditions, position of the contour $B=B_{s}$ has been established rather well. The empirical model of Fairfield (1968) yields its dayside (stand-off) distance as $\sim 11 \mathrm{R}_{\mathrm{E}}$, and a nightside distance of $\sim 7 \mathrm{R}_{\mathrm{E}}\left(\mathrm{R}_{\mathrm{E}}\right.$ is the Earth's radius). Petrinec et al. (1993) obtained a slightly different value, of about $10 \mathrm{R}_{\mathrm{E}}$, for the average stand-off distance. Behaviour of the contour during storms has not been studied thoroughly. The stand-off distance is known to decrease with geomagnetic activity. During intense storms it achieves 5-6 $\mathrm{R}_{\mathrm{E}}$ and even less (Hamilton et al., 1988; Allen et al., 1989; Rufenach et al., 1989). However the factors affecting the distance have not been established well enough. In addition the 
position of the contour $B=B_{s}$ at midnight is uncertain. Indirect evidence of the earthward displacement of this boundary during storms is the equatorward expansion of the auroral oval. According to Feldstein and Starkov (1970) Sergeev et al. (1983) and Kirkwood and Eliasson (1990), the equatorward edge of the auroral oval practically coincides with the outer boundary of the stable trapping region.

Arykov et al. (1996) and Maltsev et al. (1996) examined the dependence of the radius of the stable trapping region on storm intensity for a strongly idealized model of the magnetosphere. In this study we try to estimate the dimensions of the stable trapping region with the use of a more realistic magnetic field model based on experimental data.

\section{Magnetic field model}

An empirical model of the equatorial magnetic field for geocentric distances $<10 \mathrm{R}_{\mathrm{E}}$ has been developed by Ostapenko et al. (1996). The magnetic field is presented as

$B_{z}=\frac{B_{\mathrm{e}}}{\rho^{3}}+s_{0}+s_{1} \rho^{2}+s_{2} \rho^{4}+a_{1} \rho \cos \varphi$,

where the z-axis is antiparallel to the Earth's dipole, $\rho$ is the cylindrical distance in $\mathrm{R}_{\mathrm{E}}, B_{\mathrm{e}}=31000 \mathrm{nT}$ is the dipole magnetic field at the Earth's equator, and $\varphi$ is the longitude ( $\varphi=0$ at noon). The coefficients $s_{0}, s_{1}$ and $s_{2}$ describe the azimuthally symmetrical part of the disturbance, and the coefficient $a_{1}$ corresponds to the azimuthally asymmetrical part. Ostapenko et al. (1996) calculated the coefficients with the least square technique by using more than 2000 magnetic field measurements in the disk $|z|<2 \mathrm{R}_{\mathrm{E}}, \rho \leq 10 \mathrm{R}_{\mathrm{E}}$ borrowed from the paper by Fairfield et al. (1994). Every coefficient was found as a combination of the three parameters: the Dst index, solar wind dynamic pressure $p$, and $z$-component of the interplanetary magnetic field (IMF). Dependence on the IMF appeared to be unimportant. Relations to the other parameters (in the ranges $30>D s t>$ $-100 \mathrm{nT}, 7>p>1 \mathrm{nPa}$ ) are the following

$$
\begin{aligned}
& s_{0}=-27+1.0 \text { Dst } \\
& s_{1}=0.63-0.022 D s t, \\
& s_{2}=-3.8 \cdot 10^{-3}+1.2 \cdot 10^{-4} D s t+4.1 \cdot 10^{-4} p, \\
& a_{1}=1.9 p^{0.6} .
\end{aligned}
$$

Here $B_{z}$ and $D s t$ are in $\mathrm{nT}, p$ in $\mathrm{nPa}$. Further we extrapolate the model to intense storm conditions (up to $D s t=-600 \mathrm{nT}$ ). Such an extrapolation becomes invalid beyond the stable trapping region where the total field calculated with the use of expressions (1) and (2) can appear to be negative. As for the boundary of the stable trapping region, the plausibility of the extrapolation is supported by agreement of our computation results with the observations.

\section{Calculation of the distance to the boundary of the inner magnetosphere}

As was mentioned already the boundary of the inner magnetosphere is determined as

$B=B_{s}$,

where $B_{s}$ is the magnetic field in the subsolar point on the magnetopause. Substitution of Eq. (1) into Eq. (3) yields the following equation

$\frac{B_{\mathrm{e}}}{\rho_{s}^{3}}+s_{0}+s_{1} \rho_{s}^{2}+s_{2} \rho_{s}^{4}+a_{1} \rho_{s} \cos \varphi=B_{s}$

where $\rho_{s}=\rho_{s}(\varphi)$ is the geocentric distance to the stable trapping boundary. Equations (2) and (4) give $\rho_{s}$ as a function of Dst and $p$. The subsolar magnetic field $B_{s}$ may be obtained from the pressure balance condition

$B_{s}=\sqrt{2 \mu_{0} p_{s}}$

where $p_{s}$ is the solar wind pressure at the stagnation point. According to the calculations of Spreiter et al. (1966), we assume

$p_{s} \approx 0.88 p$.

Substitution of Eq. (6) into Eq. (5) yields

$B_{s} \approx 47 \sqrt{p}$

where $B_{s}$ is in $\mathrm{nT}, p$ in $\mathrm{nPa}$.

Equation (4) describes a curve similar to a circle with the centre shifted sunward relative to the Earth. Figure 1 shows the geocentric distances to this boundary in the dayside and nightside sectors versus $D s t$ for three values of $p(2,4$, and $15 \mathrm{nPa})$. The discontinuity of the curve in Fig. 1a for $p=2 \mathrm{nPa}$ is a consequence of the drawback of the magnetic field model, (Eq. (1) and (2)) which gives three values $\rho_{s}(D s t)$ at noon for $D s t<-200 \mathrm{nT}$. Nevertheless one can see the general tendency to erosion of the stable trapping region when the storm-time depression and solar wind pressure grow, both factors influencing the size of the region to an approximately equal extent. The erosion occurs in all the local time sectors.

\section{Mapping the boundary of the stable trapping region to the ionosphere}

The divergence-free magnetic field may be presented as

$\mathbf{B}=[\nabla \alpha \times \nabla \beta]$

where $\alpha$ and $\beta$ are Euler potentials. They are constant along magnetic field lines. Stern (1967) obtained approximate expressions for $\alpha$ and $\beta$ in the model including three fields only: that of the Earth's dipole, a homogeneous field, and the first azimuthally asymmetrical harmonic. Thus, the terms with $s_{1}$ and $s_{2}$ in the righthand side of Eq. (1) were neglected. It is not difficult to generalize the formulas of Stern (1967) with these terms being taken into account. As a result we have 

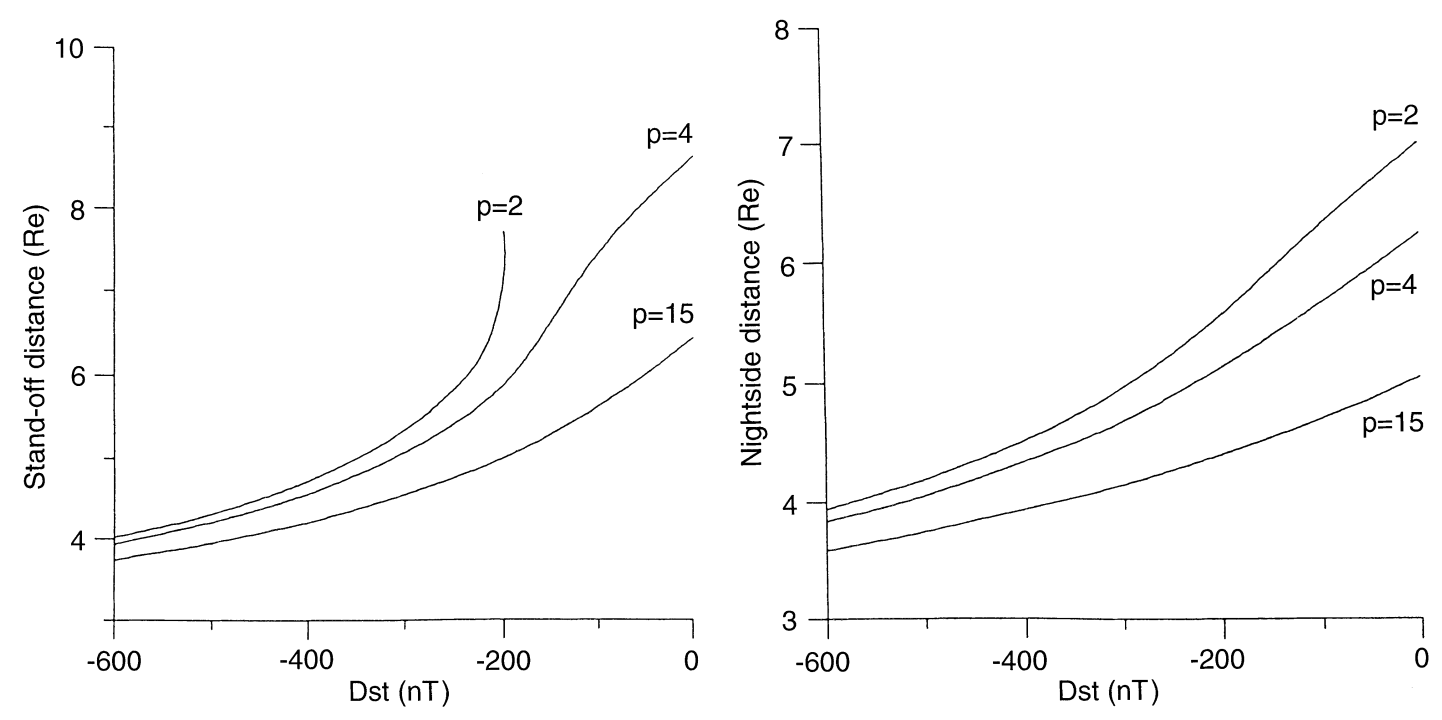

Fig. 1. Dependence of the geocentric distance to the contour $B=B_{s}$ at noon (left panel) and midnight (right panel) on the Dst index for three values of the solar wind dynamic pressure

$$
\begin{aligned}
\alpha= & \frac{B_{\mathrm{e}} \sin ^{3} \theta}{\rho}-s_{0} \frac{\rho^{2}}{2}-s_{1} \frac{\rho^{4}}{4}-s_{2} \frac{\rho^{6}}{6} \\
& +2 a_{1} \rho^{3}\left(\frac{1}{7 \sin ^{2} \theta}-\frac{1}{3}\right) \cos \varphi \\
\beta= & -\varphi+\frac{1}{7} \frac{a_{1}}{B_{\mathrm{e}}} \rho^{4} \frac{\sin \varphi}{\sin ^{5} \theta}
\end{aligned}
$$

where $\theta$ and $\varphi$ are the colatitude and longitude respectively. The distance in Eqs. (8)-(10) is in $R_{E}$, the magnetic fields in $\mathrm{nT}$. Since $\alpha=$ const along a magnetic field line, expression (9) allows us to find the latitude $\Lambda$ where the line meets the Earth if we know where it crosses the equatorial plane. On the Earth's surface $(\rho=\sin \theta=\cos \Lambda)$, the first term in the right-hand side of expression (9) is dominant and the other terms may be neglected. As a result we have for the midnight $(\varphi=\pi)$ meridian

$$
\cos ^{2} \Lambda=\frac{1}{\rho}-\frac{1}{B_{\mathrm{e}}}\left(s_{0} \frac{\rho^{2}}{2}+s_{1} \frac{\rho^{4}}{4}+s_{2} \frac{\rho^{6}}{6}-\frac{8}{21} a_{1} \rho^{3}\right)
$$

Equations (2), (4), (7), and (11) yield the dependence of $\Lambda$ on Dst shown in Fig. 2 with solid lines. The dashed line shows the latitude of the equatorward edge of the auroral oval in the nightside sector according to the empirical formula of Starkov (1993)

$$
\Lambda=74.9-8.6 \log _{10}|D s t|
$$

One can see that the calculated and empirical curves are rather close.

Note that formulas (9) and (10) are a lowest-order approximation. They give the field (1) if we neglect the terms proportional to the products $s_{0} a_{1}, s_{1} a_{1}, s_{2} a_{1}$, and $a_{1}^{2}$. In addition, formulas (9) and (10) admit some arbitrariness because they are three-dimensional whereas the model field (1) is two-dimensional. The latter does not lead to large inaccuracy because the position of the point where the magnetic field line meets the Earth's surface depends mainly on the magnetic fields in the equatorial plane of the magnetosphere and on the Earth. In an axially symmetrical field (under $a_{1}=0$ ), this may be easily obtained from the condition of the magnetic flux conservation. The azimuthal asymmetry (when $a_{1} \neq 0$ ) somewhat complicates the problem of mapping. In order to test formulas (9) and (10), we mapped the midnight part of the contour $B=B_{s}$ numerically with the use of the three-dimension field model where the external field components $B_{x}$ and $B_{y}$ equal zero, and $B_{z}$ is expressed by Eq. (1). The position of the solid curves appeared to change by not more than $0.1^{\circ}$. Thus, the Euler potentials (9) and (10) provide rather accurate mapping for distances of $<7 R_{\mathrm{E}}$.

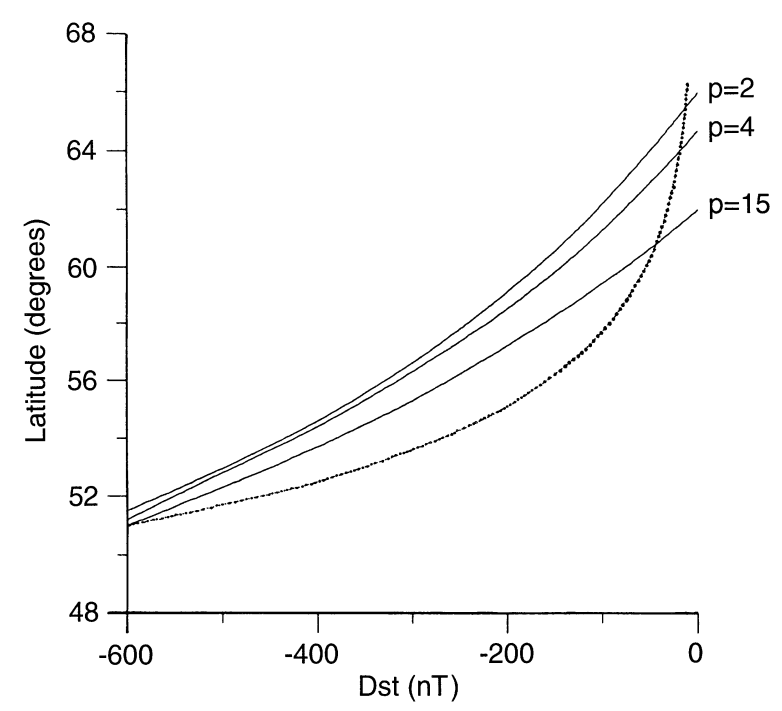

Fig. 2. Dependence of latitudes of various boundaries on the Dst index in the midnight sector. The solid lines correspond to the ionospheric projection of the contour $B=B_{s}$. The dashed line shows the storm-time behaviour of the equatorward edge of the auroral oval 


\section{Discussion}

If there were no hot plasma in the magnetosphere the stand-off distance would be as follows (Mead, 1964)

$r_{s}^{\text {Mead }}=r_{s a}\left(p_{a} / p\right)^{1 / 6}$

where $r_{s a}$ is the stand-off distance under the average solar wind pressure $\left(p=p_{a}\right)$. Petrinec et al. (1993) obtained $r_{s a}=10 \mathrm{R}_{\mathrm{E}}$. Average pressure for the data used by Ostapenko et al. (1996) was $p_{a}=2.2 \mathrm{nPa}$. During the strongest storm of the twentieth century that occurred on March 13, 1989, when Dst reached -599 nT, the stand-off distance was $4.7 \mathrm{R}_{\mathrm{E}}$ (Allen et al., 1989). During the February 8-9, 1986, storm the satellite crossed the dayside magnetopause at 5.2 $\mathrm{R}_{\mathrm{E}}$ (Hamilton et al., 1988). $D s t=-257 \mathrm{nT}$ was observed at that time. These values quite agree with the curves in Fig. 1a, although the calculated stand-off distances are somewhat smaller than the observed ones.

Rufenach et al. (1989) studied 64 magnetopause crossings of a geosynchronous satellite $\left(r=6.6 \mathrm{R}_{\mathrm{E}}\right)$. The stand-off distance $r_{s}$ was not established precisely because the crossings in the whole dayside sector from 06 to $18 \mathrm{LT}$ were examined. Since the subsolar point is closer to the Earth than any other point on the magnetopause we may conclude that the average $r_{s}$ was smaller than $6.6 \mathrm{R}_{\mathrm{E}}$. Average Dst for the 64 crossings was $-108 \mathrm{nT}$, the average solar wind dynamic pressure $p$ was $15 \mathrm{nPa}$. Formula (13) for such $p$ predicts $r_{s}^{\text {Mead }} \approx 7.9 \mathrm{R}_{\mathrm{E}}$. Figure 1a yields $r_{s} \approx 5.6 \mathrm{R}_{\mathrm{E}}$ which is not at variance with the observations.

The physical cause of the erosion of the stable trapping region is the enhancement of the electric currents flowing on the magnetopause and in the magnetotail. The magnetopause currents grow with increasing of the solar wind pressure. The cross-tail current intensifies during storms due to the magnetic flux transport from the dayside magnetosphere to the magnetotail (Dungey, 1961). The cross-tail current together with the closure currents on the magnetopause is able to depress the magnetic field in the inner magnetosphere by tens and hundreds of nanoteslas (Maltsev, 1991; Belova and Maltsev, 1994; Maltsev et al., 1996; Alexeev et al., 1996). Arykov et al. (1996) used a rather rough model of the magnetic field in the inner magnetosphere, assuming $s_{0}=\frac{2}{3} D s t, s_{1}=s_{2}=a_{1}=0$, instead of Eq. (2). The outer boundary of the stable trapping region appeared to be a concentric circle with the radius (in $\mathrm{R}_{\mathrm{E}}$ )

$\rho_{s}=\left[B_{\mathrm{e}} /\left(B_{s}-\frac{2}{3} D s t\right)\right]^{1 / 3}$

Expression (14) yields a value which is very close to the azimuthally averaged radius calculated from Eq. (4) with the use of empirical relations Eq. (2). The difference does not exceed $10 \%$ for plausible values of $B_{s}$.

A cross-tail current causes the magnetic flux erosion in the inner magnetosphere in all the local time sectors. Region 1 Birkeland currents also lead to the erosion but in the dayside sector only. According to the estimates of Maltsev and Lyatsky (1975), the Region 1 current of 3.5
MA produces the disturbance $\delta B_{z}=-17 \mathrm{nT}$ on the dayside at a distance of $10 \mathrm{R}_{\mathrm{E}}$, shifting the magnetopause from 10 to $9 \mathrm{R}_{\mathrm{E}}$. Sibeck (1994) studied signatures of the erosion observed at a geostationary satellite and found good agreement with these estimates. Since a storm is not a very frequent phenomenon the statistical relations obtained by Sibeck (1994) may be more relevant to periods without storms. Pudovkin (1982) analyzed 15 magnetopause crossings during storms and found that the magnetic field near the magnetopause was depressed compared to that predicted by the model of Mead (1964), the depression $\delta B_{z}$ reaching $-140 \mathrm{nT}$. During 64 storms investigated by Rufenach et al. (1989) the field $\delta B_{z} \leq-100 \mathrm{nT}$ of the cross-tail and Birkeland currents was necessary to remove the magnetopause from the distance $r_{s}^{\text {Mead }} \approx 7.9 \mathrm{R}_{\mathrm{E}}$ predicted by Eq. (13) for $p=15 \mathrm{nPa}$, to the observed distance $r_{s} \leq 6.6 \mathrm{R}_{\mathrm{E}}$. One can hardly expect that the value of the Region 1 Birkeland currents exceed 3.5 MA considerably, hence the contribution of the cross-tail current and the closure currents on the magnetopause to the erosion seems to dominate during storms.

Additional information about the currents responsible for the erosion can be obtained from studying the time of response to the IMF southward turning. This time is 10-20 minutes for the Region 1 Birkeland currents (Maltsev and Lyatsky, 1975) and several hours for the magnetotail currents (Maltsev et al., 1996). Rufenach et al. (1989) found that the magnetopause reached a distance of $6.6 \mathrm{R}_{\mathrm{E}}$ in average $7 \mathrm{~h}$ after the southward IMF turning. Kuznetsov et al. (1993) also pointed out that the time of response of the dayside polar cleft to the IMF southward component was not less than one hour.

The equatorward shift of the auroral oval during storms (Akasofu and Chapman, 1972; Meng, 1984; Starkov, 1993) is more evidence of the erosion of the stable trapping region. Remember that the equatorward edge of the auroral oval practically coincides with the outer boundary of the stable trapping region (Fledstein and Starkov, 1970; Sergeev et al., 1983; Kirkwood and Eliasson, 1990). Thus the most probable reason for the equatorward shift of the oval is the enhancement of the cross-tail current.

\section{Conclusion}

The inner magnetosphere outlined by the contour $B=B_{s}$ and containing the bulk of steadily trapped particles suffers erosion during both the geomagnetic storm intensification and solar wind pressure enhancement. The erosion occurs in all the local time sectors. At noon the distance to the inner magnetosphere boundary (the stand-off distance) can decrease from $\sim 10 \mathrm{R}_{\mathrm{E}}$ during quiet conditions $(D s t=0, p=2 \mathrm{nPa})$ to $\sim 4 \mathrm{R}_{\mathrm{E}}$ during very intense storms $(D s t=-600 \mathrm{nT})$. At midnight during quiet time the boundary lies at the distance of $\sim 7 \mathrm{R}_{\mathrm{E}}$. A solar wind pressure peak of $15 \mathrm{nPa}$ (under $D s t=0$ ) leads to its displacement to the distance of $\sim 5 \mathrm{R}_{\mathrm{E}}$. A storm with $D s t=-600 \mathrm{nT}$ (under $p=2-$ 
$15 \mathrm{nPa}$ ) can remove it to $\sim 4 \mathrm{R}_{\mathrm{E}}$. Ionospheric projection of the contour $B=B_{s}$ is sensitive to the Dst rather than to the solar wind pressure, with its latitude dependence on the Dst being similar to that of the equatorward edge of the auroral oval. The physical reason for the erosion is the intensification of the electric currents on the magnetopause and in the magnetotail. The auroral oval shifts equatorward due to the growth of the magnetotail currents.

Acknowledgements. This work was supported by the Russian Basic Research Foundation, grant 96-05-64305. We thank Dr. A. A. Ostapenko for the help in computations. We are grateful to the referee for constructive comments.

Topical Editor K.-H. Glaßmeier thanks M. Schulz for his help in evaluating this paper.

\section{References}

Akasofu, S.-I., and S. Chapman, Solar-terrestrial physics, Clarendon Press, Oxford, 1972.

Alexeev, I. I., E. S. Belenkaya, V. V. Kalegaev, Y. I. Feldstein, and A. Grafe, Magnetic storms and magnetotail currents, $J$. Geophys. Res., 101, 7737-7747, 1996.

Allen, J., H. Sauer, L. Frank, and P. Reiff, Effect on March 1989 solar activity, EOS Trans. AGU, 70, 1488, 1989.

Arykov, A. A., and Y. P. Maltsev, Direct-driven mechanism for geomagnetic storms, Geophys. Res. Lett., 23, 1689-1692, 1996.

Arykov, A. A., E. G. Belova, B. B. Gvozdevsky, Y.P. Maltsev, and V. V. Safargaleev, Geomagnetic storm as a result of growth of the high- latitude magnetic flux, Geomagn. Aeron., 36 (3), 39-49, 1996.

Belova, E. G., and Y. P. Maltsev, Supplementary sources of geomagnetic depression during the geomagnetic storm of 8-9 February, 1986, J. Atmos. Terr. Phys., 56, 1011-1015, 1994.

Dungey, J. W., Interplanetary magnetic field and the auroral zones, Phys. Rev. Lett., 6, 47-48, 1961.

Fairfield, D. H., Average magnetic field configuration of the quiet magnetosphere, J. Geophys. Res., 73, 7329-7338, 1968.

Fairfield, D. H., N. A. Tsyganenko, A.V. Usmanov, and M. V. Malkov, A large magnetosphere magnetic field database, $J$. Geophys. Res., 99, 11319, 1994.

Feldstein, Y. I., and G. V. Starkov, The auroral oval and the boundary of closed field lines of geomagnetic field, Planet. Space Sci., 18, 501-508, 1970.

Hamilton, D. C., G. Gloeckler, F. M. Ipavich, W. Studemann, B. Wilken, and G. Kremser, Ring current development during the great geomagnetic storm of February 1986, J. Geophys. Res., 93, 14343-14355, 1988.
Kirkwood, S., and L. Eliasson, Energetic particle precipitation in the substorm growth phase measured by EISCAT and Viking, J. Geophys. Res., 95, 6025-6037, 1990.

Kuznetsov, S. N., A. V. Suvorova, and E. D. Tolstaya, Relation between the interplanetary parameters, Dst and cleft latitude, Kosm. Issled. 31 (4), 73-81, 1993.

Maltsev, Y. P., Relation of Dst-variation to magnetospheric geometry, Geomagn. Aeron., 31, 567-570, 1991.

Maltsev, Y. P., and W. B. Lyatsky, Field-aligned currents and erosion of the dayside magnetosphere, Planet. Space Phys., 23, 1257-1260, 1975.

Maltsev Y. P., A. A. Arykov, E. G. Belova, B. B. Gvozdevsky, and V.V. Safargaleev, Magnetic flux redistribution in the storm time magnetosphere. J. Geophys. Res., 101, 7697-7704, 1996.

Mead, G. D., Deformation of the geomagnetic field by the solar wind, J. Geophys. Res., 69, 1181-1195, 1964.

Meng, C. -I., Dynamic variation of the auroral oval during intense magnetic storms, J. Geophys. Res., 89, 227-235, 1984.

Ostapenko, A. A., Y. P. Maltsev, and M. V. Malkov, Empirical model of the magnetic field in the equatorial plane of the inner magnetosphere, Geomagn. Aeron., 36 (2), 131-134, 1996.

Petrinec, S. M., P. Song, and C. T. Russell, Solar cycle variations in the size and shape of the magnetosphere, J. Geophys. Res., 98, 7893-7896, 1993.

Pudovkin, M. I., Parameters of the day side magnetopause and generation of the electric field in the magnetosphere, Ann. Geophysicae., 38, 745-753, 1982.

Roederer, J. G., On the adiabatic motion of energetic particles in a model magnetosphere, J. Geophys. Res., 72, 981-992, 1967.

Rufenach, C. L., R. F. Martin, Jr., and H. H. Sauer, A study of geosynchronous magnetopause crossings, J. Geophys. Res., 94, 15,125-15,134, 1989.

Schulz M., Geomagnetically trapped radiation, Space Sci. Rev., 17, 481-536, 1975.

Sergeev, V. A., A. G. Yahnin, and R. Pellinen, Mutual localization and magnetospheric sources of energetic electron precipitation zones, diffuse and discrete auroras, in the substorm growth phase, Geomagn. Aeron., 23, 972-978, 1983.

Shabansky, V. P., Some processes in the magnetosphere, Space Sci. Rev., 12, 299-418, 1971.

Sibeck, D. G., Signatures of flux erosion from the dayside magnetosphere, J. Geophys. Res., 99, 8513-8529, 1994.

Spreiter, J. R., A. L. Summer, and A. Y. Alksne, Hydromagnetic flow around the magnetosphere, Planet. Space Sci., 14, 223-253, 1966.

Starkov, G. V., Planetary morphology of auroras, In monograph Magnetosphere-Ionosphere Physics. Brief Handbook, Nauka., St- Petersburg, 85-90, 1993.

Stern, D. P., Geomagnetic Euler potentials, J. Geophys. Res., 72, 3995-4005, 1967. 